Vietnam Journal of Mechanics, NCST of Vietnam Vol. 22, 2000, No 4 (193 - 204)

\title{
AN IDENTIFICATION ALGORITHM FOR FEEDBACK ACTIVE CONTROL
}

\author{
NGUYEN DONG ANH \\ Institute of Mechanics, NCST of Vietnam
}

\begin{abstract}
The aim of the paper is to present a control law for feedback active controlled structures in which a control algorithm is proposed to identify the external excitation with a time delay. The time duration in which the external excitation acts on the structure is devided into subintervals. In each subinterval the external excitation is identified and is then selected with the opposite sign as the control force for the next subinterval. The realization of the identification control algorithm in the practical application mainly depends on the computing capacity of the involved computer and requires an investigation with respect to its robustness and stabilization.
\end{abstract}

\section{Introduction}

In recent years much progress and new concepts have been archieved in the development of structural control in reducing the response during excessive vibrations due to environmental loading Casciati et al. [2], Housner et al. [6], Kobori [7]. The control of structural motions can be done by various means. Among them passive and active control systems represent fundamental approaches in the use of control strategies for response reduction in structures. The first approach proposes to reduce the energy of the environmental disturbances acting on the structure by transferring its part into other structural elements (secondary systems), while the latter uses the control counterforces produced by actuators to balance the environmental loading. Since in the first approach the structure does not receive any additional energy, the application of passive devices is usually effective up to a certain limit. A major disadvantage of the second approach is that in many cases of practical interest, the required additional forces are too large existing actuators (for example, the case of earthquake excitation). Thus, the third approach which presents the combination use of passive and active control systems leads to so called hybrid control systems as an adequate means of structural protection with a high level of performance against dynamic hazards.

Since the objective is to control the structural response and external disturbances are a main source producing it, one may expect that the control problem 
would be more simplified if both structural response variables and external disturbances could be measured during the structural motion. Thus, the case where the information on both the structural response quantities and external excitations are used for designing the control forces, leads to so called feedback-feedforward (or closed-open loop) active control.

In the classical active control the active control forces can be used not only to reduce the structural response but they can be chosen in an optimal way Yao [18], Soong [14]. It seems that the active control problem for linear structures can be solved perfectly if there is not the following situation: it is usually that one is unable to measure the external excitation while the structural response can often be measured. Hence, it is obvious there is a need to look for new control laws to overcome this deficiency of classical optimal active control algorithms as above described. Many new and interesting control strategies and concepts have been proposed, such as reliability-based control, Spencer et al. [16], bounded state control, Lee and Kozin [9], Del Grosso and Zucchini [3], parametric and predictive control, Lai and Wang [8], Rodellar et al. [13], adaptive control based on the intelligent devices using fuzzy logic and neural networks, Casciati, Faravelli and Yao [1], Faravelli and Venini [4], Fu [5], Widrow and Lehr [17]. The aim of the paper is to present a control law for feedback active controlled structures. A control algorithm is proposed to identify the external excitation with a time delay.

\section{Problem formulation}

Suppose that the feedback active controlled structures is modeled by an $p$ degree of freedom system whose equation of motion is described by the nonlinear differential equation

$$
\mathbf{M} \ddot{\mathbf{z}}(t)+\mathbf{G}(\dot{\mathbf{z}}(t), \mathbf{z}(t))=\mathbf{u}(t)+\mathbf{f}(t),
$$

where $\mathbf{M}$ is the $p \times p$ mass matrix, $\mathbf{z}(t)=\left(z^{1}(t), z^{2}(t), \ldots, z^{p}(t)\right)$ is the $p \times \mathbb{1}$ displacement vector, $\mathbf{G}$ is a nonlinear function vector of displacement and velocity components, $\mathbf{f}(t)=\left(f^{1}(t), f^{2}(t), \ldots, f^{p}(t)\right)$ is the $p \times 1$ vector representing the external excitation. Since the feedback active control is considered, thus, the $p \times 1$ control force vector provided by actuators $\mathbf{u}(t)$ is selected as a function of measured response. As the structural response mainly depends on the structural parameters characterizing the internal dynamics of the structure and on the external disturbance, we consider first the structure free of external excitation and control force

$$
\mathbf{M} \ddot{\mathbf{z}}(t)+\mathbf{G}(\dot{\mathbf{z}}(t), \mathbf{z}(t))=0 .
$$


The free vibration of the system (2.2) is described as

$$
\mathbf{z}(t)=\mathbf{z}_{0}\left(t, \mathbf{z}\left(t_{0}\right), \dot{\mathbf{z}}\left(t_{0}\right)\right)
$$

where $\mathbf{z}\left(t_{0}\right), \dot{\mathbf{z}}\left(t_{0}\right)$ are initial displacement and velocity vectors of the structure. Suppose that the structure is designed in such a way that one has

$$
\left\|\mathbf{z}_{0}\left(t, \mathbf{z}\left(t_{0}\right), \dot{\mathbf{z}}\left(t_{0}\right)\right)\right\|<\delta_{1}
$$

if

$$
\left\|\mathbf{z}\left(t_{0}\right)\right\| \text { and }\left\|\dot{\mathbf{z}}\left(t_{0}\right)\right\|<\varepsilon
$$

where $\|\cdot\|$ is a norm, $\delta_{1}$ and $\varepsilon$ are small positive numbers. To archieve (2.4) one may use methods of increasing the energy dissipation capacity to reduce the amplitudes of free vibration of the structure. At present, many passive energy dissipation devices, such as friction sliding and metallic yield dampers have been installed in structures in order to enhance energy dissipation in structural systems Soong and Dargush [15]. In particular, one may use a part of the active control force in the form of linear velocity feedback

$$
\mathbf{u}_{f}(\dot{\mathbf{z}}(t))=-S \dot{\mathbf{z}}(t)
$$

for increasing damping in the structure. In terms of the stability theory it is supposed that the equilibrium position of the system $(2.2), z=0, \dot{z}=0$ is stable in Lyapunov sense. Under this assumption the control problem for the system (2.1) is to find the active control force $\mathbf{u}(t)$ necessary to keep the displacement norm below specified bounds in the presence of any disturbances less than a certain value provided that the initial values of the displacement and velocity norms are sufficiently small. In the mathematical description it means that

$$
\begin{aligned}
& \text { find } \mathbf{u}(t)=\mathbf{u}(t, \mathbf{z}(t), \dot{\mathbf{z}}(t), \ddot{\mathbf{z}}(t)) \text { such that }\|\mathbf{z}(t)\|<\delta \\
& \text { if }\left\|\mathbf{z}\left(t_{0}\right)\right\|, \quad\left\|\dot{\mathbf{z}}\left(t_{0}\right)\right\|<\varepsilon_{1} \text { and }\|\mathbf{f}(t)\|<\varepsilon_{2} .
\end{aligned}
$$

Since the undisturbanced structure is stable it is seen obviously that the best control law here would be that

$$
\mathbf{u}(t)=-\mathbf{f}(t)
$$

Indeed with the control law (2.8) the external excitation is totally eliminated. However, as it has been mentioned above the external excitation is unknown a priori, so the best control law (2.8) can not be realized in the practical application. The idea involved in the control law (2.8), however, may be used in a modified 
way. Although that the history of the external excitation can not calculated or measured in real time it can be identified piecewise with a time delay. It will be shown later the control law can be defined as

$$
\mathbf{u}(t)=-\mathbf{f}(t-\Delta)
$$

where $\Delta$ is the time delay due to the response measurement and computation of control law. In this case the sum of external excitation and control force takes the form

$$
\mathbf{f}(t)+\mathbf{u}(t)=\mathbf{f}(t)-\mathbf{f}(t-\Delta) .
$$

It seems that if $\Delta$ is sufficiently small the total force acting on the structure will be small too. More detail will be discussed later.

\section{Control algorithm}

In this section a control algorithm will be constructed to show how the history of the external excitation can be identified with a time delay. For convenience let in the equation (2.1) $\mathbf{M}=\mathbf{I}$ where $I$ is the unit matrix. Let $T$ be the time duration of action of external excitation. Further, let all components of the displacement vector can be measured during the structural motion and all components of velocity and acceleration vectors can be calculated in a short time from the corresponding components of the displacement vector as their first and second derivatives. The interval $[0, T]$ is devided into $n$ small equal intervals of the length $\Delta$ where $\Delta$ is a small positive number whose value will be discussed later. Thus, one has

$$
T=n \Delta .
$$

For any given function vector, $\mathbf{m}(t)$ the following notation is introduced:

$$
\mathbf{m}_{k}(t)=\left\{\begin{array}{lc}
\mathbf{m}(t) & (k-1) \Delta \leq t \leq k \Delta \\
0 & \text { othewise }
\end{array} \quad k=1,2, \ldots, n .\right.
$$

The control force $\mathbf{u}(t)$ will be constructed in every time subinterval $T_{k}=$ $[(k-1) \Delta \leq t \leq k \Delta]$ as follows:

In $T_{1}=[0 \leq t \leq \Delta]$, let

$$
\mathbf{u}_{1}(t)=0 .
$$

The structural response is described by the following system

$$
\ddot{\mathbf{z}}_{1}(t)+\mathbf{G}\left(\dot{\mathbf{z}}_{1}(t), \mathbf{z}_{1}(t)\right)=\mathbf{f}_{1}(t) .
$$


In the subinterval $T_{1}$ the displacement vector is measured and velocity and acceleration vectors are calculated, thus, the external excitation can be determined from (3.4):

$$
\mathbf{f}_{1}(t)=\ddot{\mathbf{z}}_{1}(t)+\mathbf{G}\left(\dot{\mathbf{z}}_{1}(t), \mathbf{z}_{1}(t)\right)
$$

In $T_{2}=[\Delta \leq t \leq 2 \Delta]$, let

$$
\mathbf{u}_{2}(t)=-\mathbf{f}_{1}(t-\Delta) \equiv-\left\{\ddot{\mathbf{z}}_{1}(t-\Delta)+\mathbf{G}\left(\dot{\mathbf{z}}_{1}(t-\Delta), \mathbf{z}_{1}(t-\Delta)\right)\right\} .
$$

The structural response is described by the following system

$$
\ddot{\mathbf{z}}_{2}(t)+\mathbf{G}\left(\dot{\mathbf{z}}_{2}(t), \mathbf{z}_{2}(t)\right)=\mathbf{u}_{2}(t)+\mathbf{f}_{2}(t) .
$$

In the subinterval $T_{2}$ the displacement vector is measured and velocity and acceleration vectors are calculated. The control force is given by (3.6). Thus, the external excitation can be calculated from (3.7):

$$
\mathbf{f}_{2}(t)=\ddot{\mathbf{z}}_{2}(t)+\mathbf{G}\left(\dot{\mathbf{z}}_{2}(t), \mathbf{z}_{2}(t)\right)-\mathbf{u}_{2}(t)
$$

or

$$
\begin{aligned}
\mathbf{f}_{2}(t) & =\ddot{\mathbf{z}}_{2}(t)+\ddot{\mathbf{z}}_{1}(t-\Delta)+\mathbf{G}\left(\dot{\mathbf{z}}_{2}(t), \mathbf{z}_{2}(t)\right)+\mathbf{G}\left(\dot{\mathbf{z}}_{1}(t-\Delta), \mathbf{z}_{1}(t-\Delta)\right) \\
\text { In } T_{k} & =[(k-1) \Delta \leq t \leq k \Delta], \text { let } \\
\mathbf{u}_{k}(t) & =-\mathbf{f}_{k-1}(t-\Delta) \\
& \equiv-\sum_{s=1}^{k-1}\left\{\ddot{\mathbf{z}}_{s}(t-(k-s) \Delta)+\mathbf{G}\left(\dot{\mathbf{z}}_{s}(t-(k-s) \Delta), \mathbf{z}_{s}(t-(k-s) \Delta)\right)\right\}
\end{aligned}
$$

The structural response is described by the following system

$$
\ddot{\mathbf{z}}_{k}(t)+\mathbf{G}\left(\dot{\mathbf{z}}_{k}(t), \mathbf{z}_{k}(t)\right)=\mathbf{u}_{k}(t)+\mathbf{f}_{k}(t) .
$$

In the subinterval $T_{k}$ the displacement vector is measured and velocity and acceleration vectors are calculated. The control force is given by (3.10). Thus, the external excitation can be calculated from (3.11):

$$
\mathbf{f}_{k}(t)=\ddot{\mathbf{z}}_{k}(t)+\mathbf{G}\left(\dot{\mathbf{z}}_{k}(t), \mathbf{z}_{k}(t)\right)-\mathbf{u}_{k}(t)
$$

or

$$
\left.\mathbf{f}_{k}(t)=\sum_{s=1}^{k}\left\{\ddot{\mathbf{z}}_{s}(t-(k-s) \Delta)+\mathbf{G}\left(\dot{\mathbf{z}}_{s}(t-(k-s) \Delta), \mathbf{z}_{s}(t-k-s) \Delta\right)\right)\right\} .
$$


In $T_{n}=[(n-1) \Delta \leq t \leq n \Delta]$, let

$$
\begin{aligned}
\mathbf{u}_{n}(t) & =-\mathbf{f}_{n-1}(t-\Delta) \\
& \equiv-\sum_{s=1}^{n-1}\left\{\ddot{\mathbf{z}}_{s}(t-(k-s) \Delta)+\mathbf{G}\left(\dot{\mathbf{z}}_{s}(t-(k-s) \Delta), \mathbf{z}_{s}(t-(k-s) \Delta)\right)\right\}
\end{aligned}
$$

The structural response is described by the following system

$$
\ddot{\mathbf{z}}_{n}(t)+\mathbf{G}\left(\dot{\mathbf{z}}_{n}(t), \mathbf{z}_{n}(t)\right)=\mathbf{u}_{n}(t)+\mathbf{f}_{n}(t) .
$$

In the subinterval $T_{n}$ the displacement vector is measured and velocity and acceleration vectors are calculated. The control force is given by (3.14). Thus, the external excitation can be calculated from (3.15):

$$
\mathbf{f}_{n}(t)=\ddot{\mathbf{z}}_{n}(t)+\mathbf{G}\left(\dot{\mathbf{z}}_{n}(t), \mathbf{z}_{n}(t)\right)-\mathbf{u}_{n}(t)
$$

or

$$
\mathbf{f}_{n}(t)=\sum_{s=1}^{n}\left\{\ddot{\mathbf{z}}_{s}(t-(k-s) \Delta)+\mathbf{G}\left(\dot{\mathbf{z}}_{s}(t-(k-s) \Delta), \mathbf{z}_{s}(t-(k-s) \Delta)\right)\right\} .
$$

Since $T_{n}$ is the last time subinterval of the time duration external excitation the control algorithm is terminated here.

\section{Mathematical proof of the control algorithm}

The mathematical proof of the control algorithm proposed can be set up as follows. Suppose the free vibration of the structural system (2.1) satisfied the condition (2.4) prove that if time delay $\Delta$ is sufficiently small the control force (3.10)

$$
\mathbf{u}_{k}(t)=-\mathbf{f}_{k-1}(t-\Delta), \quad \text { or } \quad \mathbf{u}(t)=-\mathbf{f}(t-\Delta)
$$

will yield

$$
\|\mathbf{z}(t)\| \leq \delta .
$$

provided some conditions. It is seen that this mathematical problem is serious and is thus not solved in the paper. However, some discussions will be given here for marking the control strategy more clear. The proposed control idea is based on the intuitive consideration that the small external excitation will produce from the structure stable at the equilibrium position only small vibrations if there is no resonance or other similar mechanisms. The proposed control force can balance 
the external excitation if the latter has uniform bounded derivative on the time interval $[0, T]$ and the time delay $\Delta$ is sufficiently small. In fact, denoting

$$
\mathbf{b}(t)=\mathbf{u}(t)+\mathbf{f}(t)=\mathbf{f}(t)-\mathbf{f}(t-\Delta)
$$

one has

$$
\|\mathbf{b}(t)\|=\|\mathbf{f}(t)-\mathbf{f}(t-\Delta)\|=\left\|f^{\prime}(\mathbf{s}) \Delta\right\|=\Delta\left\|f^{\prime}(\mathbf{s})\right\| \leq \varepsilon
$$

if

$$
\left\|f^{\prime}(\varsigma)\right\|<\alpha, \text { and } \Delta<\varepsilon / \alpha,
$$

where $\alpha$ and $\varepsilon$ are positive values, $\zeta$ is a $p \times 1$ constant vector and $\|\boldsymbol{\zeta}\|<\Delta$. Further, consider the case where the structure can be modeled as a linear system with bounded impulse response function $h(t)$. This requirement is satisfied if the linear system is stable. If $\delta_{1}$ and $\varepsilon$ are sufficiently small one has

$$
\begin{aligned}
\|\mathbf{z}(t)\| & =\left\|\mathbf{z}_{0}\left(t, \mathbf{z}\left(t_{0}\right), \dot{\mathbf{z}}\left(t_{0}\right)\right)+\int_{t_{0}}^{t} h(s)\{\mathbf{u}(t-s)+\mathbf{f}(t-s)\} d s\right\| \\
& \leq \| \mathbf{z}_{0}\left(t, \mathbf{z}\left(t_{0}\right), \dot{\mathbf{z}}\left(t_{0}\right)\left\|+\int_{t_{0}}^{t}\right\| \mathbf{h}(s) \mathbf{b}(t-s) \| d s\right. \\
& \leq \delta_{1}+\int_{t_{0}}^{t}\|h(s)\|\|\mathbf{b}(t-s)\| d s \leq \delta_{1}+\beta \varepsilon T \leq \delta
\end{aligned}
$$

if $\delta_{1}$ and $\varepsilon$ are sufficiently small positive numbers, $\beta$ is the bound of the impulse response function

$$
\|\mathbf{h}(t)\|<\beta .
$$

Thus, one has proved that if the linear structure is stable the control algorithm proposed can keep the structural response smaller than any bound provided that the initial displacement and velocity vectors and time delay $\Delta$ are sufficiently small.

\section{Use of parallel computing}

For practical application the identification control algorithm proposed should be transferred into-computer control algorithm which is then implemented in a software. As it has been seen in the previous section the effectiveness of the identification control algorithm essentially depends on the value of the time delay 
subinterval $\Delta$ in which on-line measurement and computation are conducted in every step. As smaller the value $\Delta$ smaller the controlled structural response. Thus, the problem of how to minimize the computation real time in the computer control algorithm is needed to investigate. In this context, recent remarkable progresses in computing technology may be used to solve the problem. In fact, the use of modern computing facilities allows to explore problems which were totally impractical earlier. In particular, when serial computers reach fundamental limits in performance parallel computers may be used to increases computing execution speed and accuracy. Parallel computers have been utilized in many field of engineering and in particular in control, see Proceedings of First World Conference on Parallel Computing in Engineering [12], Pai [11], Marsi et al. [10]. To investigate the possibility of use of parallel computing to solve our control problem let consider again the identification control algorithm. At each time subinterval once the displacement vector is known from measurement the following computations are required:

a) Calculation of all components of velocity and acceleration vectors, namely,

$$
\dot{z}^{1}(t), \dot{z}^{2}(t), \ldots, \dot{z}^{p}(t) \text { and } \ddot{z}^{1}(t), \ddot{z}^{2}(t), \ldots, \ddot{z}^{p}(t)
$$

b) Calculation of all components of external excitation, namely, $f^{1}(t)$, $f^{2}(t), \ldots, f^{p}(t)$

It is obvious that all the computation requirements involved in a) and b) can straightforward be conducted in parallel computing. Thus, the parallel computing block can be defined as follows. Suppose the computer has $m$ processors: $P_{1}, P_{2}, \ldots, P_{m}$, the computation distribution and the information exchange between them are following:

1) Processor $P_{i}$ computes $\dot{z}^{K_{i}}(t)$ and $\ddot{z}^{K_{i}}(t)$ for $(i-1)[p / m] \leq K_{i} \leq i[p / m]$, $i=1,2, \ldots, m$.

2) All processors exchange their calculation results to each other

3) Processor $P_{i}$ computes $f^{K_{i}}(t)$ for $(i-1)[p / m] \leq K_{i} \leq i[p / m]$, $i=1,2, \ldots, m$.

The identified external excitation in the current time subinterval will be selected with opposite sign as the control force for the next time subinterval.

\section{Numerical simulation}

For the numerical simulation we consider a single degree of freedom structure subjected to deterministic excitation. Thus, the external excitation force is taken 
in a sinusoidal form:

$$
f(t)=\sin 5 t+0.1 \sin 23 t .
$$

The equation of motion of the structure is described by the following second order differential equation

$$
\ddot{\mathbf{z}}(t)+2 \zeta \omega \dot{\mathbf{z}}(t)+\omega^{2} \mathbf{z}(t)=\mathbf{u}(t)+\mathbf{f}(t),
$$

where $\zeta=0.03$ and $\omega=14$. The time duration of action of the external excitation is $[0,5]$. The identified algorithm for feedback active control is used to reduce the response of the system (6.2). The corresponding time-delay subinterval is taken as $\Delta=0.05$ ( $1 \%$ of the time duration of action) and the control force after the identification procedure has the form $\mathbf{u}(t)=-\mathbf{f}(t-\Delta)$. The result is shown in Fig. 1 where two curves are presented: one with points is uncontrolled response

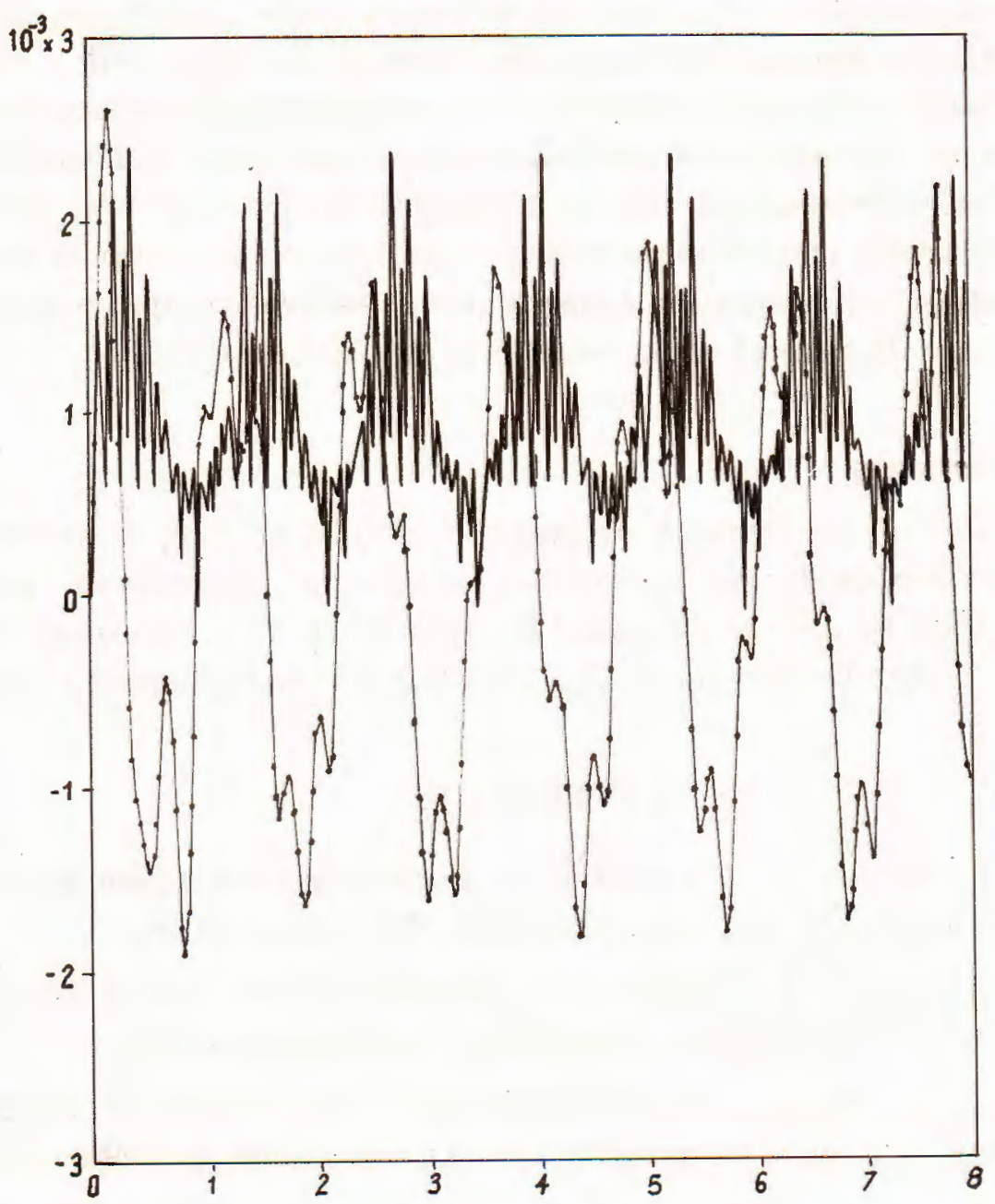

Fig. 1. Numerical simulation

a) the curve with points is uncontrolled response, b) the continuous curve is controlled response 
and another (continuous line) is controlled response. It is seen that the magnitude of the controlled response is equal approximately a half of the magnitude of the uncontrolled response. The controlled effect could be better if the time delay would be smaller.

\section{Conclusion}

In recent years much progress and new concepts have been archieved in the development of the structural control in reducing the response during the excessive vibration due to environment loading such as earthquakes and winds as above described. Although many new and interesting control strategies have been proposed, there is always a need to look for new control laws to overcome the deficiency of the classical optimal active control algorithms. The aim of the paper is to present a control law for feedback active controlled structures in which a control algorithm is proposed to identify the external excitation with a time delay. The time duration in which the external excitation acts on the structure is devised into subintervals. In each subinterval the external excitation is identified and is then selected with the opposite sign as the control force for the next subinterval. The realization of the identification control algorithm in the practical application mainly depends on the computing capacity of the involved computer and requires an investigation with respect to its robustness and stabilization.

\section{Acknowledgements}

The support of the research program in natural sciences is acknowledged. The paper was written during the author's stay at the University of Pavia which was made possible by a CICOPS award for 1999-2000. The discussion with Prof. Casciati is thankful. Author thanks Dr. Breitung for the numerical simulation.

\section{REFERENCES}

1. Casciati F., Faravelli L., Torelli G. A Fuzzy Chip controlled for nonlinear vibrations approach. Nonlinear Dynamics, 20 (1999), 85-98.

2. Casciati F., Maceri F., Singh M. P., Spanos P. (Eds.) Civil infrastructure systems: intelligent renewal, World Scientific, Singapore 1998.

3. Del Grosso A., Zucchini A. Bounded state active control of structures: a set-theoretic approach, Smart Material and Structures, 4 (1995), A15-A24.

4. Faravelli L., Venini P. Active structural control by neutral networks, J. of Structural Control, 1, N 1-2 (1994), 79-102. 
5. Fu K. S. Learning control systems and intelligent control systems: an intersection of artifical intelligence and automatic control, IEEE Trans. on Automatic Control, 16 (1971), 70-72.

6. Housner G. W., Bergman L. A., Caughey T. K., Chassiakos A. G., Claus R. O., Marsi S. F., Skelton R. E., Soong T. T., Spencer B. F., and Yao J. T. P. Structural control: past, present and future, ASCE, J. Engrg. Mech., 123 (9) (1997), 897-958.

7. Kobori T. Future direction on research and development of seismic-response controlled structure, Proc., First World Conf. on Struct. Control (1994), 19-31.

8. Lai J. S., Wang K. W. Parametric control of structural vibration via adaptive stiffness. J. of Vibration and Acoustics, 118 (1996), 41-47.

9. Lee S. K., Kozin F. Bounded state control of structures with uncertain parameters, ASCE, Dynamics Response of Structures, (1986), 788-794.

10. Marsi S. F., Bekey G. A., Caughey T. K. and Van de Velde E. Adaptive stochastic optimization using multiprocessors, Appl. Math. and Computation, 72 (1995), 225-257.

11. Pai D. K. Programming parallel distributed control of complex systems, Proc. IEEE Int. Symposium on Intelligent Control, Albany, New York, 1989.

12. Proceedings of First World Conf. on Parallel Computing in Engineering and Engneering education, (1990) UNESCO, Paris, October 8-12, The Microcomputer Unit Ltd, London.

13. Rodellar J., Barbat A. H., and Martin-Sanchez D. K. Predictive control of structures, ASCE, J. Engrg. Mech., 113 (1986), 797-817.

14. Soong T. T. Active structural control: Theory and practice, John Wiley and Sons, Inc., New York, 1990.

15. Soong T. T., Dargush G. F. Passive Energy Dissipation systems in structural enginnering, John Wiley and Sons, Inc. New York 1997.

16. Spencer B. F., Jr. Kaspari D. C. and Sain M. K. Structural control design: a reliability-based approach, Proc. Am. Control Conf. (1994), 1062-1066.

17. Widrow B. and Lehr M. A. Thirty years of adaptive neural network: perceptron, madaline and backpropagation. Proc. IEEE, 78 9, (1990). 1415-1441.

18. Yao J. T. P. Concepts of structural control, ASCE 98, J. Struct. Div. (1972), 1567-1574.

Received July 30,2000 
Bài báo trình bày luật điều khiển cho hệ kết cấu được điều khiền theo cách tích cực phản hồi, theo đó một thuật toán điều khiển được đề nghị nhằm xác định ngoại lực với một sự chậm về thời gian. Khoảng thời gian trong đó ngoại lực tác động lên hệ kết cấu được chia làm $n$ khoảng nhỏ. Tại mỗi khoảng thời gian nhỏ ngoại lực được xác định và sau đó sẽ được chọn với dấu ngược lại để làm lực điều khiển cho khoảng thời gian nhỏ tiếp theo. Sự áp dụng thuật toán điều khiển trên trong thực tế phụ thuộc vào khà năng và tốc độ của máy tính được cài đặt trong hệ kết cấu. Trong mối quan hệ đó việc sử dụng kỹ thuật tính song song đã được đề cập như một phương tiện rút ngắn thời gian tính toán.

Institute of Mechanics, 264 Doi Can, Hanoi, Vietnam

Email: ndanh@im01.ac.vn . 